\title{
Performance characteristic of endoscopic ultrasound- guided fine needle aspiration is unaffected by pancreatic mass size
}

Authors

Institutions
Jayapal Ramesh' ${ }^{1}$, Hwasoon Kim², Kartika Reddy ${ }^{1}$, Isam-Eldin A. Eltoum ${ }^{3}$

${ }^{1}$ Division of Gastroenterology and Hepatology, University of Alabama at Birmingham, Birmingham, Alabama, USA

${ }^{2}$ Department of Biostatistics, University of Alabama at Birmingham, Birmingham, Alabama, USA

${ }^{3}$ Department of Pathology, University of Alabama at Birmingham, Birmingham, Alabama, USA submitted 17. August 2015 accepted after revision 25 . January 2016

\section{Bibliography}

Dol http://dx.doi.org/

10.1055/s-0035-1569969

Published online: 30.3.2016

Endoscopy International Open 2016; 04: E434-E438

(c) Georg Thieme Verlag KG

Stuttgart · New York

E-ISSN 2196-9736

\section{Corresponding author}

Jayapal Ramesh, MD

Basil Hirschowitz Endoscopic

Center of Excellence

Division of Gastroenterology and Hepatology

University of Alabama at

Birmingham

BDB 389

1808 7th Avenue South

Birmingham

Alabama

35294

USA

Fax: +1-205-975-6381

j1ramesh@gmail.com
Background and study aims: Despite a well-established tool for diagnosis of pancreatic masses, endoscopic ultrasound-guided fine needle aspiration (EUS-FNA) studies have shown suboptimal diagnostic performance at divergent mass sizes. Since the impact of gold standard follow-up and presence of on-site evaluation on this observation is unknown, we aimed to study the performance characteristics of EUS-FNA under these strict conditions.

Patients and methods: EUS-FNA results from pancreatic mass lesions performed between July 2000 and March 2013 were evaluated. All patients with histological follow-up were then stratified into four groups: Group A ( $\leq 10 \mathrm{~mm})$, Group B (11-20mm), Group C (21-40mm), and Group D $(>40 \mathrm{~mm})$. Sensitivity and diagnostic accuracy were calculated for each group and compared. Results: A total of $612 / 3832(16 \%)$ patients with pancreatic masses who underwent EUS-FNA had

\section{Introduction}

\section{$\nabla$}

Endoscopic ultrasound (EUS) is a well-established, cost-effective procedure for investigating solid pancreatic mass lesions [1,2]. While CT scan is the primary modality for investigating pancreatic disease, EUS is the preferred tool in highly suspicious circumstances due to its ability to reveal lesions in the absence of a mass on CT scan [3] and its superior performance characteristics for diagnosing lesions less than $30 \mathrm{~mm}$ in size [4]. Between 2006 and 2010, the usage of endoscopic ultrasound-guided fine needle aspiration (EUS-FNA) increased by $69.3 \%$; percutaneous biopsy increased by $1.8 \%$ compared to a corresponding decrease in open surgical biopsy by $41.7 \%$ [1]. With the growing utilization of EUSFNA [5], there is a renewed focus to evaluate and target small lesions as early stage diagnosis leads to more curative resections and significantly impacts clinical outcomes. histology confirmation. Of these, 81 were excluded due to unavailable lesion size, while the rest formed the study cohort. Mean age (SD) was 65.8 years (9.3) with $51.2 \%$ female. The mean number of passes for the entire cohort was 2.9 (SD 1.9; range $1-12$ ); patients in group $D$ had a significantly higher number of passes for on-site diagnosis $(P=0.0124)$. There was no significant difference between the groups for sensitivity $(P=$ $0.1134)$ or diagnostic accuracy $(P=0.2111)$. Proportional trend analysis revealed no significant correlation between size and sensitivity $(P=$ 0.6192). The size of lesion measured by EUS was not associated with sensitivity or specificity after adjusting for age, sex, and pancreatic location. Conclusion: In the presence of rapid on-site cytopathology and when final histology is taken as the gold standard, pancreatic mass size does not affect the performance characteristics of EUS-FNA.

Although EUS-FNA has a high sensitivity and specificity for diagnosis of pancreatic mass lesions, diagnostic accuracy of EUS-FNA is dependent on operator experience [6], presence of rapid on-site cytopathologist evaluation (ROSE) [7], needle size [8], and the presence of chronic pancreatitis [9]. In the presence of a mass and high clinical suspicion but a negative EUS-FNA, there is also evidence that repeat EUS-FNA improves diagnostic accuracy [10]. Even though a recent meta-analysis confirmed that diagnostic accuracy is superior in the presence of ROSE [7], suboptimal EUS-FNA performance was noted for pancreatic mass sizes at either end of the spectrum. A study from 1999 [11] showed that the sensitivity of EUS-FNA for small lesions was modest while another study showed that diagnostic accuracy rises as the size of the mass increases with a reduction in accuracy for lesions more than $40 \mathrm{~mm}$ [12]. While additional endoscopic ultrasound imaging features may aid in the differentiation between solid pan- 


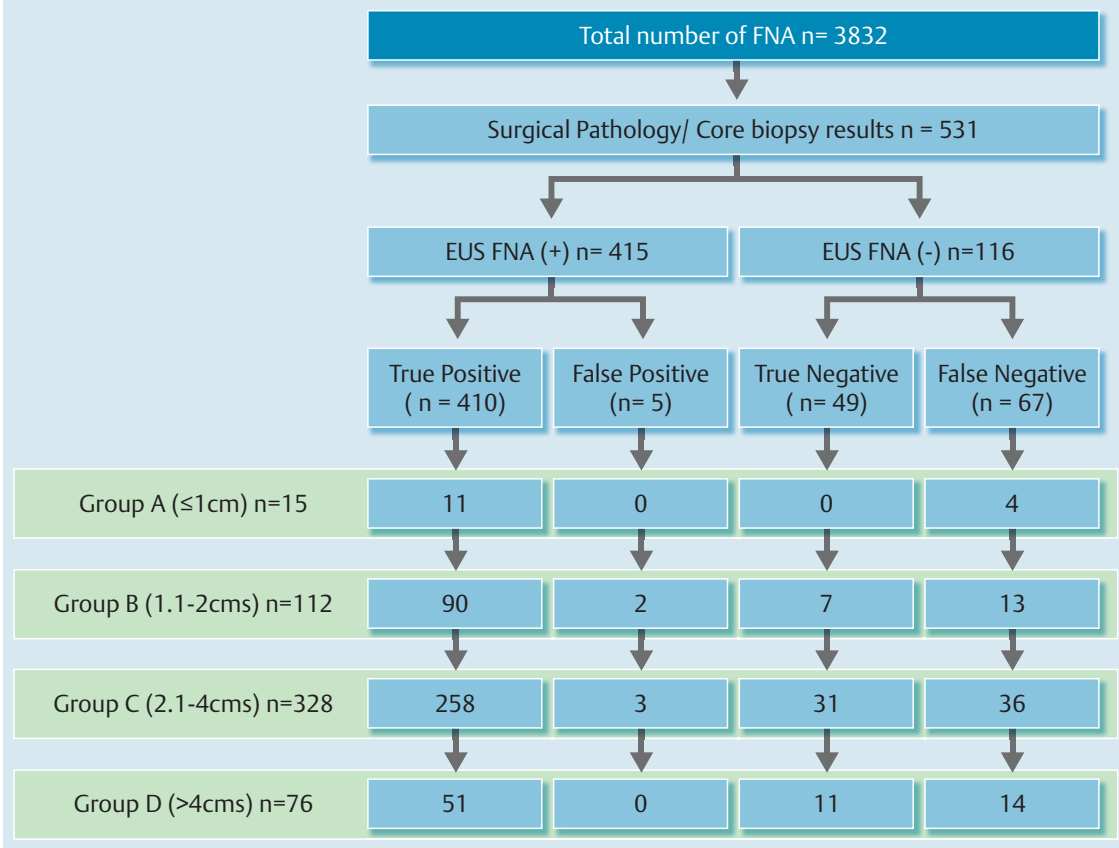

Fig. 1 Flow chart showing the included study subjects.

creatic mass lesions [13] and are helpful for predicting the possibility of adenocarcinoma [14], tissue diagnosis is essential for further clinical management.

Given the importance of pancreatic mass size for staging, increasing use of EUS-FNA for tissue acquisition and uncertainty with regard to its performance at different mass sizes, we sought to study the operating characteristics of EUS-FNA vis-à-vis pancreatic mass size under gold standard conditions.

\section{Materials and methods}

\section{Patient population}

This is a retrospective clinicopathologic correlation study of patients who underwent EUS-FNA of pancreatic masses from July 2000 to March 2013. Data including EUS findings, rapid on-site cytopathology, and final histology were documented in an institutional review board (IRB) approved database. After identifying all patients who had final histology as the gold standard for diagnosis, the available cohort was stratified into four groups based on longest dimension of cross-sectional pancreatic mass sizeGroup A ( $\leq 10 \mathrm{~mm})$, Group B (11 - $20 \mathrm{~mm})$, Group C (21 - $40 \mathrm{~mm})$, and Group D ( $>40 \mathrm{~mm}$ ). These four groups were compared for the operating characteristics of EUS-FNA.

\section{Procedure}

EUS was performed under moderate sedation or anesthesia with a linear array echoendoscope (Olympus UCT140, Olympus America Corp, Center Valley, PA, United States) utilizing the standard station approach by four experienced endosonographers. When a mass was identified, cross-sectional size measurements were undertaken along with echo features, surrounding vasculature, evaluation for peripancreatic lymph nodes, and examination of the liver. Fine needle aspiration (FNA) of the mass was performed with a standard FNA needle (Echotip, Cook Endoscopy, WinstonSalem, NC, United States; Expect ${ }^{\mathrm{TM}}$, Boston Scientific Corporation, Natick, MA, United States); the size of the needle (25G, 22G, and $19 \mathrm{G}$ ) used was determined by individual choice of the endosono- grapher. Patients then recovered as per standard procedure in the endoscopy unit and were discharged when stable.

\section{Preparation of specimen for on-site analysis}

Predominantly air-dried and a few alcohol-stained smears were prepared on-site after individual passes. Air-dried smears were stained with Diff-Quick stain (Baxter, McGraw Park, IL, United States) for immediate review by a cytopathologist to ascertain sample adequacy and provide a preliminary diagnosis. Number of passes made was dependent on on-site evaluation for adequacy and diagnosis. In the cytopathology laboratory, alcoholstained smears were prepared using Papanicolaou's stain; cell block pellets were prepared, sectioned, and stained with routine hematoxylin and eosin. The cytopathologist then characterized the diagnosis into previously described [15] cytopathologic categories: positive for malignancy; negative for malignancy; atypical; suspicious; benign and non-diagnostic after additional review of slides.

\section{Preparation of cellblock for histological analysis}

The EUS-FNA specimen was placed in Cytolyte and taken to the laboratory where it was spun in the centrifuge. After decanting the supernatant, the sediment was made into a pellet and placed in a Tissue-Loc HistoScreen cassette (Microm International, Walldorf, Germany) and fixed in formalin. Thereafter, it was embedded in paraffin and sections made for hematoxylin and eosin (H\&E) staining to examine for the presence of a histological core. Only the diagnostic specimens with definable histological core were included.

\section{Surgical pathology specimens}

Once surgery was performed, surgical pathology specimens were analyzed and the final diagnosis recorded in the database. Patients who had histological core tissue acquired at the time of surgical exploration, but were deemed inoperable were also included in the database. Surgically obtained specimens were sectioned and placed in formalin. These were further processed, embedded in paraffin, and stained with hematoxylin and eosin. 
Table 1 Patient characteristics and EUS findings for the entire cohort.

\begin{tabular}{|c|c|c|c|c|c|}
\hline & Group A $(n=15)$ & Group B $(n=112)$ & Group C $(n=328)$ & Group D ( $n=76)$ & $P$ value \\
\hline Age, mean (SD), years & $65(4)$ & $67(7)$ & $67(9)$ & $64(12)$ & 0.0564 \\
\hline Sex, n (\%) & & & & & 0.8811 \\
\hline Male & $6(40)$ & $56(50)$ & $165(50)$ & $37(49)$ & \\
\hline Female & $9(60)$ & $56(50)$ & $163(50)$ & $39(51)$ & \\
\hline Location, n (\%) & & & & & 0.0002 \\
\hline Uncinate & - & $14(13)$ & $37(11)$ & $5(7)$ & \\
\hline Head & $9(60)$ & $70(62)$ & $196(60)$ & $32(42)$ & \\
\hline Body & $4(27)$ & $20(18)$ & $39(12)$ & $12(16)$ & \\
\hline Tail & $2(13)$ & $8(7)$ & $56(17)$ & $27(35)$ & \\
\hline Number of passes, mean (SD) & $2.2(1.7)$ & $3(2.1)$ & $2.8(1.8)$ & $3.5(1.9)$ & 0.0124 \\
\hline Final diagnosis, n (\%) & & & & & $<0.0001$ \\
\hline Adenocarcinoma & $7(47)$ & $79(70)$ & $225(69)$ & $28(37)$ & \\
\hline Neuroendocrine tumor & $4(27)$ & $15(13)$ & $24(7)$ & $6(8)$ & \\
\hline Lymphoma & - & $1(1)$ & $9(3)$ & $8(10.5)$ & \\
\hline Chronic pancreatitis & - & $6(5)$ & $31(9)$ & $8(10.5)$ & \\
\hline Others & $4(26)$ & $11(11)$ & $39(12)$ & $26(34)$ & \\
\hline
\end{tabular}

Table2 Operating characteristics of EUS-FNA between the groups.

\begin{tabular}{|llllll|}
\hline & Group A & Group B & Group C & Group D & Total \\
\hline Sensitivity & 0.7333 & 0.8738 & 0.8776 & 0.7846 & 0.8595 \\
\hline Diagnostic accuracy & 0.7333 & 0.8661 & 0.8811 & 0.8158 & 0.8644 \\
\hline
\end{tabular}

\section{Statistical analysis}

Lesions defined as "malignant" and "suspicious" by EUS-FNA, with a final pathology diagnosis of malignancy, were categorized as True Positive (TP) for applying the Multinominal Logistic Regression (MLR) model; those with benign final diagnosis were categorized as False Positive (FP). Similarly, patients diagnosed as "negative" and "benign" by histology were considered True Negative (TN), whereas those "negative" or "atypical" on EUS-FNA but malignant on surgical pathology were categorized as False Negative (FN). In all of these, only the final cytologic diagnosis was considered in the analysis. Chi-squared tests were used for each categorical variable (sex, location of the mass, final diagnosis, sensitivity) to test their association among the four groups. Analysis of variance (ANOVA) was conducted to compare means of age and the number of FNA passes across all groups. Cochran-Armitage trend tests were performed to examine for increasing or decreasing trends in diagnostic accuracy and sensitivity with tumor size. Pairwise comparisons of absolute difference among the groups were done by Chi-squared tests with Bonferroni adjustment.

As suggested by Dwivedi et al. [16], we fitted multinomial logistic regression models for the four outcomes (TP, FP, TN, and FN) with covariates of age, sex, diameter of lesion as measured by EUS, and pancreatic location. We considered two separate models with maximum and minimum diameter of lesion per outcome category. The sensitivity model used false negative as reference value and the specificity model used false positive as reference value. Two-sided $P$ values $<0.05$ were considered statistically significant. Analysis was done using SAS software, version 9.3 (SAS Institute Inc., Cary, NC, United States), as well as IBM-SPSS version 22 (Armonk, New York, United States).

\section{Results}

$\nabla$

A total of 3832 EUS-FNAs were performed during the study period. Of these, 612 patients had histological follow-up. Accurate information with regard to mass size was lacking in 81 cases and these were excluded. The rest (531/3832; 13.9\%, 95\%CI: 12.8 15.0) formed the study cohort ( $\mathbf{~ F i g . 1 ) . ~}$

\section{Demographics}

The groups were evenly matched for age and gender, while there was a significant difference in the location of the mass with predominant lesions noted in the head of pancreas $(P=0.0002)$. Final diagnosis revealed a significant proportion of ductal adenocarcinoma $(P<0.0001)$. The results are shown in $\bullet$ Table 1 .

\section{Operating characteristics of EUS-FNA}

The overall sensitivity was $85.95 \%$, diagnostic accuracy was $86.44 \%$ and with a positive predictive value of $98.8 \%$ ( $\odot$ Table 2 ). The mean number passes of 2.2 (SD 1.7), 3 (SD 2.1), 2.8 (SD 1.8), and 3.5 (SD 1.9) required for on-site diagnosis $(\boldsymbol{O}$ Fig. 2 ) between the four groups was statistically significantly different $(P=$ $0.0124)$. There was no significant relationship between the four groups and sensitivity $(P=0.1134)$. The Cochran-Armitage trend test did not reveal an increasing or decreasing trend in diagnostic accuracy $(P=0.9923)$ or the probability of finding a true positive result in relation to tumor size $(P=0.6192)$.

As shown in $\bullet$ Table 3 and $\bullet$ Table 4 , the maximum diameter of lesion measured by EUS was not associated with sensitivity or specificity after adjusting for age, sex, and pancreatic location. We observed similar results from the models with the minimum diameter of lesion (not presented). The models for positive predictive value (PPV) and negative predictive value (NPV) showed 


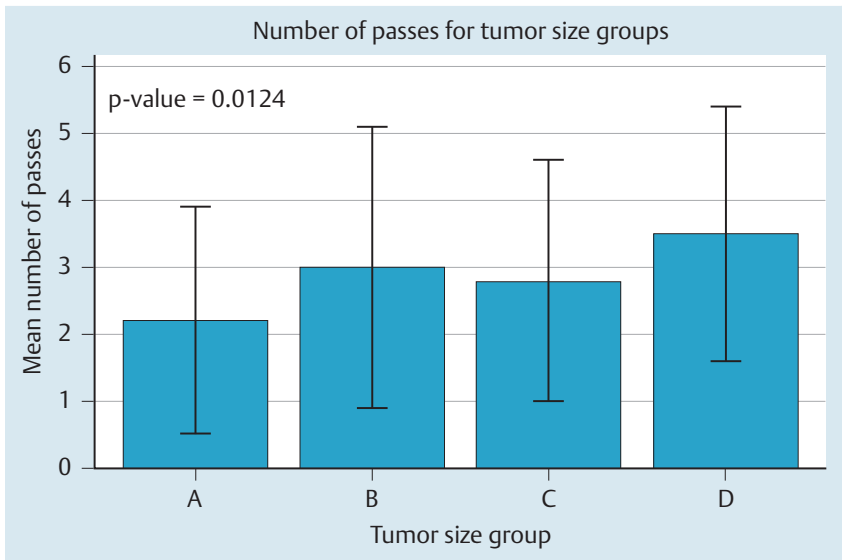

Fig. 2 Bar chart depicting the mean number of passes required for on-site diagnosis between the four groups.

similar results in terms of significance of the size of lesion (not presented).

\section{Discussion}

This study determined that, under controlled, gold standard conditions, the performance of EUS-FNA is unaffected by pancreatic mass size and confirmed that, to obtain an on-site diagnosis, an increasing number of passes is required for larger lesions.

Siddiqui et al. [12] showed that diagnostic accuracy and sensitivity were strongly correlated with tumor size with a significant reduction for tumors less than $10 \mathrm{~mm}$; however, in that study, the number of cases in each group was not reported, on-site assessment was rendered by a cytotechnician, and follow-up criteria included clinical review. In another multicenter study, Sahai et al. [11] concluded that the image quality and/or depth of penetration were insufficient to permit successful FNA of smaller lesions. However, that study was performed with a mechanical sectorscanning transducer without Doppler capability, not all centers that participated in the study had rapid on-site evaluation, and
FNA passes were restricted to less than three. In contrast, our decade long experience is with curvilinear array echoendoscopes in the presence of ROSE, the number of passes made was smaller, and we used histological follow-up exclusively. In the last decade, advancements in echoendoscope technology and needle designs have allowed the endosonographer to accurately target even deeply placed small lesions with greater efficiency and accuracy. Since a small mass is a concentrated, dense area of abnormal cells with no necrosis, suitably targeted FNA often provides "excellent" material with minimal contamination. Sample adequacy is tested immediately by ROSE and, if negative, care is taken to ensure suitable needle placement inside the lesion for subsequent passes to achieve an accurate diagnosis.

Erickson et al. reported that the presence of a cytopathologist during EUS-FNA improves diagnostic yield, decreases the number of unsatisfactory samples, reduces the need for more passes, and consequently, the procedural duration [17]. Our experience reflects that study as the mean number of passes for on-site diagnosis for the whole cohort was 2.6. In a recent meta-analysis, Hebert-Magee et al. showed that the presence of ROSE increases the diagnostic accuracy of EUS-FNA for pancreatic adenocarcinoma but lower performance was seen when histology alone was taken as the gold standard while this was higher for studies with clinical follow-up [7]. This may explain the low rates noted in our data in comparison to other studies. ROSE is a vital feature as good communication, team work, and interdisciplinary collaboration are crucial for obtaining an on-site diagnosis. The presence of ROSE helps not only in reducing the number of passes, but also improving overall diagnostic accuracy [17]; that study also showed that EUS-FNA performance is unaffected by pancreatic mass size.

In a randomized trial of 54 patients with solid pancreatic mass lesions, the fanning technique established a significantly higher first pass diagnosis in $85.7 \%$ of patients compared to only $57.7 \%$ with the standard technique [18]. That study highlighted the importance of technique as a key factor for successful tissue procurement. It is well documented that larger lesions tend to have necrotic material in the center of the lesion and targeting the periphery yields adequate tissue for diagnosis [19]. Our data reflect other previously noted observations $[12,20]$ that multiple passes

\begin{tabular}{|llll|}
\hline & Adjusted odds ratio (OR) & P value & 95\%Cl for adjusted OR \\
\hline Age & 1.01 & 0.543 & $0.97-1.03$ \\
\hline Maximum diameter of lesion & 0.94 & 0.546 & $0.78-1.14$ \\
\hline Female & 0.96 & 0.890 & $0.57-1.64$ \\
\hline Pancreatic location & 1 & & \\
\hline Uncinate & 0.72 & 0.509 & $0.27-1.93$ \\
\hline Head & 0.61 & 0.419 & $0.19-2.00$ \\
\hline Body & 1.27 & 0.698 & $0.38-4.26$ \\
\hline Tail & & & \\
\hline
\end{tabular}

Table 3 Parameter estimates for sensitivity model with maximum diameter of lesion.

\begin{tabular}{|llll|}
\hline & Adjusted odds ratio (OR) & P value & 95\%Cl for adjusted OR \\
\hline Age & 0.97 & 0.401 & $0.89-1.05$ \\
\hline Maximum diameter of lesion & 2.01 & 0.187 & $0.70-6.14$ \\
\hline Female & 0.64 & 0.649 & $0.09-4.43$ \\
\hline Pancreatic location* & & & \\
\hline Body & 0.66 & 0.771 & $0.04-11.06$ \\
\hline Head & 3.36 & 0.370 & $0.24-47.56$ \\
\hline Uncinate & 1 & & \\
\hline
\end{tabular}

Table 4 Parameter estimates for specificity model with maximum diameter of lesion. 
are required for larger lesions to make a diagnosis. However, randomized data in the presence of ROSE and the fanning technique indicate that on-site diagnosis can be achieved in a majority with a single pass $[17,21,22]$. Another randomized trial comparing 19G vs. 25G FNA needles also showed that on-site diagnostic adequacy can be achieved in more than $97 \%$ with a single pass even in large pancreatic lesions [23]. These studies also showed that, in the presence of ROSE, when a structured fanning technique is adopted, diagnosis can be achieved in a significant majority with a single pass independent of needle or pancreatic mass sizes. Our study had several limitations. First, the number of pancreatic masses in group A was small which may have had an impact on the results; this, however, reflects the strict histopathology criteria adopted in this study. Second, the procedure was performed by experienced endosonographers, without trainee involvement, with proficient and expert on-site cytopathologists in attendance; therefore, the results may not be applicable to all units and if trainees were involved. Third, only patients who underwent index FNA were included in the analysis while patients who underwent repeat EUS-FNA for high clinical suspicion were excluded; the results may be vastly different if these were included in the analysis. Fourth, there is a likelihood of verification bias within the dataset that precluded complete specificity analysis. Fifth, needle size and pathology subcategory stratification analysis could not be performed due to small size and inconsistent data availability. Finally, this is a retrospective study and has its attendant inadequacies; however, unlike other studies of EUSFNA, the strength of this study lies in the stringent criteria used for follow-up.

In conclusion, this study shows that in the presence of ROSE, pancreatic mass size does not affect the performance of EUS-FNA, even when final histology is taken as gold standard. This further emphasizes the effectiveness of obtaining an on-site diagnosis.

\section{Competing interests: None}

\section{References}

1 Roy AK, Kim M, Hawes $R$ et al. 196. Changing trends in tissue acquisition in pancreatic diseases. Gastrointest Endosc 2013; 77: AB134

2 Hewitt MJ, McPhail MJ, Possamai L et al. EUS-guided FNA for diagnosis of solid pancreatic neoplasms: a meta-analysis. Gastrointest Endosc 2012; 75: 319-331

3 Wang W, Shpaner A, Krishna SG et al. Use of EUS-FNA in diagnosing pancreatic neoplasm without a definitive mass on CT. Gastrointest Endosc 2013; 78: 73-80

4 Muller MF, Meyenberger C, Bertschinger P et al. Pancreatic tumors: evaluation with endoscopic US, CT, and MR imaging. Radiology 1994; 190: $745-751$

5 Eltoum IA, Alston EA, Roberson J. Trends in pancreatic pathology practice before and after implementation of endoscopic ultrasound-guided fine-needle aspiration: an example of disruptive innovation effect? Arch Pathol Lab Med 2012; 136: 447-453

6 Eloubeidi MA, Tamhane A. EUS-guided FNA of solid pancreatic masses: a learning curve with 300 consecutive procedures. Gastrointest Endosc 2005; 61: 700-708
7 Hebert-Magee S, Bae S, Varadarajulu S et al. The presence of a cytopathologist increases the diagnostic accuracy of endoscopic ultrasoundguided fine needle aspiration cytology for pancreatic adenocarcinoma: a meta-analysis. Cytopathology 2013; 24: 159-171

8 Madhoun MF, Wani SB, Rastogi A et al. The diagnostic accuracy of 22gauge and 25-gauge needles in endoscopic ultrasound-guided fine needle aspiration of solid pancreatic lesions: a meta-analysis. Endoscopy 2013; 45: 86-92

9 Varadarajulu S, Tamhane A, Eloubeidi MA. Yield of EUS-guided FNA of pancreatic masses in the presence or the absence of chronic pancreatitis. Gastrointest Endosc 2005; 62: 728 - 736

10 Suzuki R, Lee JH, Krishna SG et al. Repeat endoscopic ultrasound-guided fine needle aspiration for solid pancreatic lesions at a tertiary referral center will alter the initial inconclusive result. J Gastrointest Liver Dis 2013; 22: 183-187

11 Sahai AV, Schembre D, Stevens PD et al. A multicenter U.S. experience with EUS-guided fine-needle aspiration using the Olympus GFUM30P echoendoscope: safety and effectiveness. Gastrointest Endosc 1999; 50: $792-796$

12 Siddiqui AA, Brown LJ, Hong SK et al. Relationship of pancreatic mass size and diagnostic yield of endoscopic ultrasound-guided fine needle aspiration. Dig Dis Sci 2011; 56: 3370-3375

13 Aso A, Ihara E, Osoegawa T et al. Key endoscopic ultrasound features of pancreatic ductal adenocarcinoma smaller than $20 \mathrm{~mm}$. Scand J Gastroenterol 2014; 49: $332-338$

14 Eloubeidi MA, Luz LP, Tamhane A et al. Ratio of pancreatic duct caliber to width of pancreatic gland by endosonography is predictive of pancreatic cancer. Pancreas 2013; 42: 670-679

15 Eloubeidi MA, Tamhane A, Jhala $N$ et al. Agreement between rapid onsite and final cytologic interpretations of EUS-guided FNA specimens: implications for the endosonographer and patient management. Am J Gastroenterol 2006; 101: 2841-2847

16 Dwivedi AK, Mallawaarachchi I, Figueroa-Casas J et al. Multinomial logistic regression approach to the evaluation of binary diagnostic test in medical research. Stat Transit New Ser 2015; 16: 1-20

17 Erickson RA, Sayage-Rabie L, Beissner RS. Factors predicting the number of EUS-guided fine-needle passes for diagnosis of pancreatic malignancies. Gastrointest Endosc 2000; 51: 184-190

18 Bang JY, Magee SH, Ramesh J et al. Randomized trial comparing fanning with standard technique for endoscopic ultrasound-guided fine-needle aspiration of solid pancreatic mass lesions. Endoscopy 2013; 45: $445-450$

19 Ekberg O, Bergenfeldt M, Aspelin P et al. Reliability of ultrasound-guided fine-needle biopsy of pancreatic masses. Acta Radiol 1988; 29: 535 539

20 Ranney N, Phadnis M, Trevino J et al. Impact of biliary stents on EUSguided FNA of pancreatic mass lesions. Gastrointest Endosc 2012; 76: $76-83$

21 Bang JY, Hebert-Magee S, Trevino J et al. Randomized trial comparing the 22-gauge aspiration and 22-gauge biopsy needles for EUS-guided sampling of solid pancreatic mass lesions. Gastrointest Endosc 2012; 76: $321-327$

22 Ramesh J, Bang JY, Hébert-Magee S et al. 1022. Multi-center randomized trial comparing the $19 \mathrm{G}$ and $25 \mathrm{G}$ needles for EUS-guided FNA of solid pancreatic mass lesions. Gastrointest Endosc 2013; 77: AB179AB180

23 Hasan M, Ramesh J, Bang JY et al. 100. Multi-center randomized trial comparing the 19G and 25G needles for EUS-guided FNA of large solid pancreatic mass lesions. Gastrointest Endosc 2014; 79: AB112 